\title{
Validity of the (WIFI) classification system in predicting the risk of amputation in patients with non healing ulcer
}

\author{
Wafi F. Salib, ${ }^{1}$ Atef A. Desokey, ${ }^{2}$ Ramez M. Wahba, ${ }^{2}$ Mohammed O. Zayed ${ }^{2}$ \\ ${ }^{1}$ Department of General Surgery, Ain Shams University, Egypt \\ ${ }^{2}$ Department of Vascular Surgery, Ain Shams University, Egypt
}

Background: Classification systems are powerful tools for health care providers to use when managing patients with threatened limbs. The ability to define and delineate a heterogeneous group into fine-grained cohorts not only aids communication between providers, but also allows for a more accurate analysis of outcomes across treatment strategies. Thus, classification systems are essential for clinical decision making as well as setting meaningful goals and expectations with patients and their families.

Aim of the work: To evaluate the predicative ability of this classification in a real world selection at Ain Shams University at period of 1 year depending on the Society for Vascular Surgery, Classification depending on: Wound, Ischemia and Foot Infection (WIFI).

Patients and methods: This study included 60 patients with non-healing wound ulcer at Ain Shams University hospitals during year 2017 and 2018.

Results: As our study showed WIfI classification was predictive of 1 year limb amputation and wound non healing and correlated significantly with outcomes predicted by the SVS consensus panel. The study showed 1 year amputation rates were $0 \%$ for stage $1,7.7 \%$ for stage 2, $18.75 \%$ for stage 3 And $64.7 \%$ for stage 4 . It also showed among the 60 patients studied 15 patients had done amputations where $6.7 \%$ were stage 2, 20\% were stage 3 and $73.7 \%$ were stage 4 .

Conclusion: WIFI classification can be very useful in predicting the possibility of amputation during 1 year; also the study showed the benefit of using WIFI to plan management of patients presented with foot ulcer.

Key words: WIFI classification system, amputation non-healing ulcer.

\section{Introduction}

Classification systems are powerful tools for health care providers to use when managing patients with threatened limbs. The ability to define and delineate a heterogeneous group into finegrained cohorts not only aids communication between providers, but also allows for a more accurate analysis of outcomes across treatment strategies. Thus, classification systems are essential for clinical decision making as well as setting meaningful goals and expectations with patients and their families. ${ }^{1}$

Old classification schemes fall short in capturing the full spectrum of disease for threatened limbs. The Fontaine and Rutherford classifications, commonly in use for threatened limbs and PAD, are purely ischemic models. Neither classification includes infection or provides sufficient detail of wound severity. ${ }^{1}$

Similarly, the widely used Wagner and University of Texas wound classification systems lack proper assessment of perfusion status and infection. The Wagner system does not account for severity of PAD nor does it delineate gangrene due to infection versus ischemia. The University of Texas system includes PAD and infection, but lacks severity gradation for either category. ${ }^{2}$

The Society for Vascular Surgery Lower Extremity Threatened Limb (SVS WIFI) classification system has three components: Wound, Ischemia and Foot Infection. Each component is graded on a spectrum from 0 (none) to 1 (mild) to 2 (moderate) to 3 (severe) based on grades assigned to each of the three individual components, a WIFI class is assigned. Each Class is categorized to certain stage: Stage 1: Amputation risk: very low, Stage 2: Amputation risk: low, Stage 3: Amputation risk: moderate, Stage 4: Amputation risk: high and Stage 5: Unsalvageable foot ${ }^{3}$ The Society for Vascular Surgery WIfI system is intended for any patient with a diabetic foot ulcer, non-healing foot ulcer present for two or more weeks, foot/lower extremity gangrene, or ischemic rest pain. It is not meant for patients with acute ischemia, emboli, trauma, nonatherosclerotic diseases such as vasospastic disorders, or pure venous ulcers.

Wound: The first category accounts for the degree 
of tissue loss and anticipated level of intervention/ amputation required for healing. ${ }^{3}$

Ischemia: The second category assesses perfusion status to the foot using objective hemodynamic indices such as ankle brachial index (ABI), transcutaneous oximetry, pulse volume recording, skin perfusion pressure or toe pressure. ${ }^{3}$

Foot infection: The last category describes the foot infection and derives from the IDSA and PEDIS clinical staging systems. ${ }^{3}$

\section{Aim of the Work}

The Society for Vascular Surgery; wound, ischemia and foot infection (WIFI) classification was proposed to predict amputation risk and potential benefit from revascularization. The goal of this study was to evaluate the predicative ability of this classification in a real world selection at Ain Shams University at a period of 1 year.

\section{Patients and methods}

Study setting: The study included patients with nonhealing wound ulcer at Ain Shams University hospitals.

Study period: Patients with non-healing wound ulcer at Ain Shams University hospitals during year 2017 and 2018.

Study population: 60 patients with non-healing wound ulcer at Ain Shams University hospitals during year 2017 and 2018.

\section{Inclusion criteria:}

The Society for Vascular Surgery WIFI system is intended for any patient with:

1. Diabetic foot ulcer.

2. Non-healing foot ulcer present for two or more weeks.

3. Foot/lower extremity gangrene, or ischemic rest pain.

\section{Exclusion criteria:}

- Critically ill patients.

- Patients with acute ischemia, emboli, trauma, non-atherosclerotic diseases such as vasospastic disorders, or pure venous ulcers.

Sampling method: Random sampling of patients Ain shams university hospitals.

Sample size: 60 patients. Ethical Considerations:

According to approved standards to Ethical Committee of Ain Shams University.

\section{Study tools}

1. Complete history taking:

- Age of presentation.

- Medical habits e.g. smoker (number of packs per day and date starting smoking), Alcohol or drug.

- intake.

- Medical history e.g. DM, HTN, IHD, history of Stroke, Renal or liver impairments.

- Cause of wound and date wound appeared at foot.

2. Clinical evaluation and examination:

- Amount of tissue loss and degree of infection.

- Depth and size of wound.

- Site of the wound.

- Discharge from wound.

- ABIusingVascularDopplerandSphygmomanometer.

\section{Arterial duplex and CT angiography on arterial tree of both lower limbs if needed.}

Statistical analysis: Data were collected, tabulated and propriety statistical analyses were applied such as Annova, t-Test, and Pearson Correlation.

Statistical package: Excel 2010, SPSS vers. 24 and N-Primer. 


\section{Results}

Table 1

\begin{tabular}{|c|c|c|}
\hline Demographics & Total no. $=60$ & \\
\hline \multirow{2}{*}{ Age } & Mean \pm SD & $59.22 \pm 10.97$ \\
\hline & Range & $39-82$ \\
\hline \multirow{2}{*}{ Sex } & Male & $37(61.7 \%)$ \\
\hline & Female & $23(38.3 \%)$ \\
\hline \multirow{2}{*}{ Smoking } & No & $48(80.0 \%)$ \\
\hline & Yes & $12(20.0 \%)$ \\
\hline \multirow{2}{*}{ Cigarettes/d } & Median (IQR) & $20(20-40)$ \\
\hline & Range & $10-60$ \\
\hline \multirow{2}{*}{ Cigarettes/m } & Median (IQR) & $600(600-1200)$ \\
\hline & Range & $300-1800$ \\
\hline \multirow{2}{*}{ Obesity } & No & $50(83.3 \%)$ \\
\hline & Yes & $10(16.7 \%)$ \\
\hline \multirow{2}{*}{ DM } & No & $0(0.0 \%)$ \\
\hline & Yes & $60(100.0 \%)$ \\
\hline \multirow{2}{*}{ HTN } & No & $29(48.3 \%)$ \\
\hline & Yes & $31(51.7 \%)$ \\
\hline \multirow{2}{*}{ Hypercholesterolemia } & No & $45(75.0 \%)$ \\
\hline & Yes & $15(25.0 \%)$ \\
\hline \multirow{2}{*}{ IHD } & No & $35(58.3 \%)$ \\
\hline & Yes & $25(41.7 \%)$ \\
\hline \multirow{2}{*}{$\mathrm{CHF}$} & No & $60(100.0 \%)$ \\
\hline & Yes & $0(0.0 \%)$ \\
\hline \multirow{2}{*}{ CVA } & No & $56(93.3 \%)$ \\
\hline & Yes & $4(6.7 \%)$ \\
\hline \multirow{2}{*}{ Renal insufficiency } & No & $54(90.0 \%)$ \\
\hline & Yes & $6(10.0 \%)$ \\
\hline
\end{tabular}

Table 2

\begin{tabular}{lll}
\hline Initial investigations & Total no. $=\mathbf{6 0}$ & \\
\hline HGB & Mean \pm SD & $11.40 \pm 1.59$ \\
& Range & $8.2-14.5$ \\
TLC & Mean \pm SD & $10.79 \pm 3.76$ \\
& Range & $5-20$ \\
PLT & Mean \pm SD & $334.15 \pm 96.94$ \\
& Range & $115-599$ \\
CRP & Median (IQR) & $8(6-18)$ \\
& Range & $3.5-96$ \\
ESR & Mean \pm SD & $74.00 \pm 32.24$ \\
& Range & $20-140$ \\
HbA1c & Mean \pm SD & $8.52 \pm 1.72$ \\
& Range & $6-13.1$ \\
Creat & Mean \pm SD & $1.16 \pm 0.95$ \\
& Range & $0.6-7.5$ \\
Alb & Mean \pm SD & $3.35 \pm 0.58$ \\
INR & Range & $2-4.5$ \\
& Mean \pm SD & $1.13 \pm 0.11$ \\
\hline & Range & $0.9-1.3$ \\
\hline
\end{tabular}


As our study showed WIfI classification was predictive of 1 year limb amputation and wound non healing and correlated significantly with outcomes predicted by the SVS consensus panel.

The study was done on 60 patients, which all had Diabetes, from Ain Shams University Hospitals during period of 2017 and 2018 from which there were 23 female $(38.3 \%)$ and 37 male $(61.7 \%)$.
The study showed that the 1 year amputation rates were $0 \%$ for stage $1,7.7 \%$ for stage $2,18.75 \%$ for stage 3 and $64.7 \%$ for stage 4 . It also showed among the 60 patients studied 15 patients had done amputations where $6.7 \%$ were stage 2, 20\% were stage 3 and $73.7 \%$ were stage 4 .

Table 3: Percentage of amputations in each stage from total amputations performed in the study

\begin{tabular}{ccc}
\hline WIFI Clinical stage & $\begin{array}{c}\text { Number of amputations done } \\
\text { during 2017 and 2018 }\end{array}$ & $\begin{array}{c}\text { Percentage form total } \\
\text { amputations }\end{array}$ \\
\hline 1 & 0 & $0 \%$ \\
2 & 1 & $6.7 \%$ \\
3 & 3 & $20 \%$ \\
4 & 11 & $73.3 \%$ \\
All stages & 15 & $100 \%$ \\
\hline
\end{tabular}

Table 4: Percentage of amputations from patients in each stage

\begin{tabular}{cccc}
\hline WIFI Clinical stage & Number of patients & $\begin{array}{c}\text { Number of } \\
\text { amputations done } \\
\text { during 2017 and 2018 }\end{array}$ & $\begin{array}{c}\text { Percentage of } \\
\text { amputations in each } \\
\text { stage }\end{array}$ \\
\hline 1 & 14 & 0 & 0 \\
2 & 13 & 1 & $7.70 \%$ \\
3 & 16 & 3 & $18.75 \%$ \\
4 & 17 & 11 & $64.7 \%$ \\
\hline
\end{tabular}

Table 5: Patients subjected or not subjected to amputation in each stage

\begin{tabular}{lccccc}
\hline Risk of amputation & $\begin{array}{c}\text { Amputation } \\
\text { Not done } \\
\text { No. }=\mathbf{4 5}\end{array}$ & $\begin{array}{c}\text { Test value* } \\
\text { Done } \\
\text { No. }=\mathbf{1 5}\end{array}$ & P-value & Sig. & \\
\hline Very low & $14(31.1 \%)$ & $0(0.0 \%)$ & 21.371 & 0.000 & HS \\
Low & $12(26.7 \%)$ & $1(6.7 \%)$ & & & \\
Moderate & $13(28.9 \%)$ & $3(20 \%)$ & & \\
High & $6(13.3 \%)$ & $11(73.3 \%)$ & & & \\
\hline
\end{tabular}

P-value $>0.05$ : Non significant; P-value $<0.05$ : Significant; P-value $<0.01$ : Highly significant *: Chisquare test.

\section{Discussions}

As our study showed WIfI classification was predictive of 1 year limb amputation and wound non healing and correlated significantly with outcomes predicted by the SVS consensus panel.

The study showed 1 year amputation rates were $0 \%$ for stage 1

$7.7 \%$ for stage 2
$18.75 \%$ for stage 3

$64.7 \%$ for stage 4

It also showed among the 60 patients studied 15 patients had done amputations where

$6.7 \%$ were stage 2

$20 \%$ were stage 3

$73.7 \%$ were stage 4 
The study shows the correlation between the 1 year risk of amputation and the stage in WIFI classification.

In comparison to the studies done by Nestoras Mathioudakis, MD, et al. J Vasc Surg 2017 which showed major amputations continued to plague the most severe stage 4 WIfI patients, with 1-year amputation rates of $20 \%$ to $64 \%$ among patients with diabetic foot ulcers (DFUs) treated in a multidisciplinary setting. ${ }^{1}$

In this study There were 217 DFU patients with 439 wounds (mean age, 58.360 .8 years; $58 \%$ male, $63 \%$ black) enrolled, including $28 \%$ WIfI stage $1,11 \%$ stage $2,33 \%$ stage 3 , and $28 \%$ stage $4 .{ }^{1}$

Minor amputations where $18 \%$ in stage 1 and $56 \%$ in stage 4 . Major amputation at 1 year. ${ }^{1}$

Moreover this study showed peripheral arterial disease and dialysis were more common in patients with advanced (stage 3 or 4 ) wounds ( $P$ \# .05). Demographics of the patients, socioeconomic status, and comorbidities were otherwise similar between groups.

This supports that the WifI classification system correlated risk of major amputation at 1 year.

However; use of a multidisciplinary approach for DFUs may augment healing time and reduce amputation risk compared with previously published historical controls of standard wound care among patients with advanced stage 4 disease.

Another study done by Joseph L. Mills Sr 2014 ElsevierInc showed three single-center studies validating the underlying premises and basic concept of the SVS WIfI Threatened Limb Classification System. ${ }^{3}$

The Greenville group recently reported the results of 158 revascularization procedures performed in 139 patients during a 3-year period and classified patients after revascularization based on the SVS WIfI classification. The WIfI clinical stage was predictive of major limb amputation and wound non

healing and correlated significantly with outcomes predicted by the SVS consensus panel. ${ }^{3}$

The reported major amputation rates were $3 \%$, $10 \%, 23 \%$ and $40 \%$ for clinical stages $1,2,3$ and 4 respectively. The corresponding wound nonhealing rates were $8 \%, 19 \%, 30 \%$ and $63 \%$ as one progressed from clinical stage1 up to clinical stage 4 .

It should be noted that this group applied the SVS WIfI classification after revascularization which would be analogous so the results most applicable to patients with more severe grades of ischemia.

The University of Arizona group prospectively applied SVS WIfI classification to a group of 201 consecutive patients presenting with threatened limbs during a 2-year period. All patients had wounds at presentation, and $93 \%$ of the total cohort had diabetes mellitus. ${ }^{3}$

WifI classifications were calculated at baseline, before initiation of any treatment, and included patients with a broad spectrum of wounds and ischemia and a significant number with infection.

No patients in clinical stage 1 and 2 required amputation, $10 \%$ of the amputations were in clinical

stage 3 patients and $90 \%$ were in clinical stage 4 .

One-year amputation free survival rates were $100 \%$ for clinical stages 1 and 2, 92\% for clinical stage 3 and dropped to $63 \%$ for clinical stage 4 .

Wound healing time was also found to correlate strongly with clinical stage, progressively increasing with each clinical stage, ranging from 94 days in clinical stage 1 to 264 days for clinical stage4. There was a significant difference between mean wound healing time of stage 1 and 3 patients ( $P 1 / 4$.048). However, the most significant delay of wound healing was in stage4 (263 days, 95\% confidence interval, 167_360) compared to clinical stages 1 to 3 (P0.002).

Revascularization resulted in a profoundly accelerated wound healing time, especially in clinical stage3 patients ( $P$ 1/4 .008).

The University of California (San Francisco) group prospectively applied SVS WifI classification to 63 threatened limbs in 50patients, $70 \%$ were diabetic. $^{3}$

No limbs in stage 1 or 2 patients required amputation, but both minor and major amputation rates increased in stage $3(19 \%$ and $6 \%)$ and stage 4 patients (59\% and $24 \%$ ), respectively (P $1 / 4.01)^{3}$

Wound score and infection score were associated with major amputation, but baseline comorbidities were not.

Ischemia score did not correlate with amputation risk in this series, but the revascularization approach was aggressive and nearly all revascularizations were successful.

Those 3 studies supported furthermore the predicitive ability of WIFI classification in determining 1 year amputation rates. Also they showed that WIFI correlated with wound non healing and how revascularization decrease the risk of amputation and time of wound healing.

Also; in 2014, Cull et al ${ }^{17}$ examined and graded 139 patients with foot wounds undergoing any lower extremity revascularization and concluded that increases in the WIfI clinical stages correlate with poorer wound healing and lower rates of 1-year limb salvage. ${ }^{4}$

Similarly, in 2015, Zhan et al evaluated 201 patients 
with threatened limbs undergoing any lower extremity revascularization, illustrating that an increase in the WIfI clinical stage increases the risk of 1-year amputation, decreases 1-year amputation-free survival, and prolongs wound healing. Our data both expand and corroborate these claims, validating the WIfI classification system for any first-time lower extremity revascularization for CLTI. ${ }^{5}$

In 2016, Beropoulis et al further validated the WifI classification system in a prospective study of 302 CLTI patients undergoing endovascular treatment. They found statistical differences in 1-year amputation and survival between clinical stages 1 and 4 but no differences between clinical stages 1 to $3 .{ }^{6}$

Our results show that WIFI classification has high accuracy in stratification and assessing the risk of amputations in patients.

This concludes the benefit of usage of WIFI classification during assement of patients with foot

ulcers and before deciding on the management and plan of treatment.

However; more studies should be further done and other co-morbidities should be taken in account during planning of management for foot ulcers. Also the general condition of the patient and whether the patient is bed ridden or active and the life style of the patient should be taken in account as it can affect the decision of amputation.

Moreover; it should be taken in account that staging of foot ulcer changes during treatment for example after revascularization or debridment. Also as seen in the study 60 patients were diabetic while the WIFI classification can include diabetic and no diabetic foot ulcers which conclude the need for futher studies on the application of WIFI classification.

\section{Conclusion}

Our prospective study shows that WIFI classification can be very useful in predicting the possibility of amputation during 1 year; also it shows the benefit of using WIFI to plan management of patients presented with foot ulcer. As a result, physicians and medical institutions should implement WIFI classification on patients presented to them before planning treatment and even during treatment of patients as patients can be restaged as shown in several studies.

\section{References}

1. Rutherford's Vascular Surgery and Endovascular Therapy, Volume 5 Set $9^{\text {th }}$ Edition; 2018 by Anton N Sidawy MD MPH (Author), Bruce A Perler MD MBA (Author).

2. Oyibo SO: A comparison of two diabetic foot ulcer classification systems. Diabetes Care. 2001; 213; 77(123): 90-99.

3. Mills JL, Conte MS: Armstrong DG, Pomposelli FB, Schanzer A, Sidawy AN, Andros G: The Society for Vascular Surgery Lower Extremily Treatment Limb Classification System: Risk stratification based on wound, ischemia and foot infection (WIFI). $\boldsymbol{J}$ Vasc Surg. 2014; 59: 220-234.

4. Lozano F, González-Porras JR, March JR, Lobos JM, Carrasco E and Ros E: Diabetes mellitus and intermittent claudication: A cross-sectional study of 920 claudicant. Diabetol Metab Syndr. 2014; 455; 90(87): 34-38.

5. Hingorani A, LaMuraglia GM, Henke $P$, et al: The management of diabetic foot: A clinical practice guideline by the Society for Vascular Surgery in collaboration with the American Podiatric Medical Association and the Society for Vascular Medicine. J Vasc Surg. 2016; 63: 3S-21S.

6. Mandolfino T, Canciglia A, Salibra M, Ricciardello D, Cuticone G: Functional outcomes of transmetatarsal amputation in the diabetic foot: Timing of revascularization, wound healing and ambulatory status. Updates Surg. 2016; [Epub ahead of print]. 